\title{
Efficiency and intensity noise of an all-fiber optical parametric oscillator
}

\author{
IoAnnis Begleris ${ }^{1}$ And Peter HoraK ${ }^{1, *}$ \\ ${ }^{1}$ Optoelectronics Research Centre, University of Southampton, Southampton SO17 1BJ, United Kingdom \\ *Corresponding author: peh@orc.soton.ac.uk
}

Compiled January 3, 2019

\begin{abstract}
An all-fiber optical parametric oscillator comprising a highly nonlinear optical fiber and wavelength division multiplexing couplers is numerically simulated. The model is based on a set of coupled nonlinear Schrödinger equations which spectrally resolve the gain bandwidth of the pump, signal and idler waves. We show that such high wavelength resolution is necessary to obtain the correct threshold pump powers, stationary output powers, and relative intensity noise. Finally, we compare seeded and unseeded configurations and find that the seeded configuration generates low-noise idler output at conversion efficiencies of up to $10 \%$ below the threshold power for unseeded operation, but that both seeded and unseeded oscillators produce comparable results above threshold. () 2019 Optical Society of America
\end{abstract}

http://dx.doi.org/10.1364/ao.XX.XXXXXX

\section{INTRODUCTION}

Fiber optical parametric amplifiers (FOPAs) have long been considered as attractive systems for wavelength conversion mainly due to the large bandwidth operation that they can potentially provide [1, 2]. However, because of the low Kerr nonlinearity of silica-based fibers, long propagation lengths are normally required to achieve sufficient gain. On the other hand, all optical fibers exhibit some non-uniformity along their lengths, such as minor diameter fluctuations, which affect the fiber dispersion properties and thus affect the phase matching condition required for efficient four-wave mixing (FWM) which forms the basis of parametric amplification. The non-uniformity of optical fibers therefore greatly reduces the efficiency of practical FOPAs [3] and can also increase the pump power thresholds [4].

One way to overcome this is to use shorter-length highly nonlinear fibers (HNLF). Significant further gain enhancement can be achieved if the fiber is used inside a ring resonator, thereby forming a fiber optical parametric oscillator (FOPO), where a coherent build-up of circulating power leads to stronger nonlinear effects.

The first FOPO system, termed the modulation instability laser, was theoretically and experimentally characterized by Nakazawa et al. [5, 6]. Experimental studies with similar apparatus have been conducted for both pulsed [7-9] and continuous wave $(\mathrm{CW})$ configurations with high conversion efficiencies [1013]. The conversion efficiency of these systems is subject to the gain of the HNLF, similar to FOPAs, but also depends on the transmittance of the signal within the FOPO and the relative phase of the waves under consideration [14].

FOPO systems have also been investigated numerically in a few studies. The most straightforward approach is to model a single amplitude for each of the waves involved, i.e., for the pump wave and the generated signal and idler waves [1]. However, this is a CW approach (and thus cannot simulate e.g. noise properties) and it requires a priori knowledge of the generated signal and idler wavelengths. Since these wavelengths depend on phase matching and thus also on nonlinear phase shifts, they will generally depend on pump power and may change during the time evolution of the system. To simulate the full temporal and spectral dynamics, an approach based on the generalized nonlinear Schrödinger equation $[15,16]$ has been exploited. The downside of this is that it becomes numerically very challenging and inefficient for large wavelength separations between the pump, signal, and idler since a continuum spectrum is effectively simulated where most wavelength components contain no power [17]. Alternatively, a set of coupled nonlinear Schrödinger equations has been used [18-20] where the three waves are described by individual time/wavelength dependent amplitudes. These models can efficiently simulate large spectral separations, but they do not take into account the Raman contribution to the nonlinearity even for signal wavelengths within the Raman gain bandwidth of the pump. Furthermore, cascaded FWM processes leading to higher order signal and idler sidebands that have been shown to increase the instability of the system [21, 22] are neglected in this approach.

In addition, beam splitters, gratings, or optical couplers are required to form the FOPO resonator. Care needs to be taken with regard to the wavelength selectivity of these components. In particular, if both the signal and the idler wave oscillate within the cavity this can lead to back-conversion of power from signal 


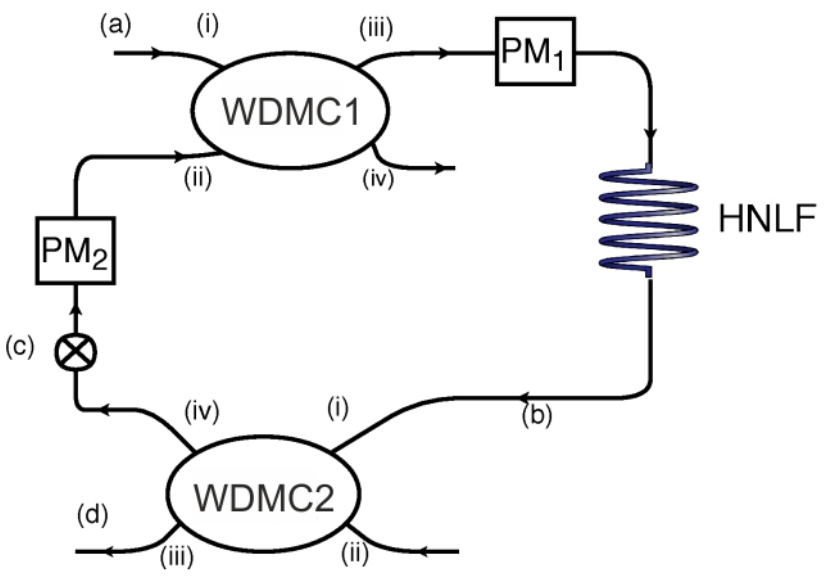

Fig. 1. Schematic of the FOPO setup comprising a highly nonlinear fiber (HNLF), two wavelength division multiplexing couplers (WDMC) for input/output and two phase modulators (PM) to control the phases of the circulating light.

and idler to the pump [23] limiting conversion efficiency and increasing the instability of the system. This instability can be reduced if only the signal is oscillated[24, 25], which also can be achieved in an all-fiber FOPO configuration using wavelength division multiplexer couplers (WDMC) [11, 13].

In this article we simulate numerically an all-fiber WDMCbased FOPO [13] using an enhanced version of the banded nonlinear Schrödinger equation (BNLSE) [17] which allows for broadband simulations with increased spectral resolution, correct treatment of the Raman nonlinearity at any wavelength separation between pump and signal, and also includes higher order signal and idler sidebands to capture cascaded FWM processes. A realistic model of the WDMCs is used to include parasitic effects induced by a residual amount of idler signal that is circulating within the resonator. We focus on $\mathrm{CW}$ pumping with and without externally seeding the signal wave and investigate the FOPO performance in terms of conversion efficiency (CE) of pump power to the idler wave output and the relative intensity noise (RIN) depending on system parameters.

\section{OPTICAL SYSTEM}

The simulated fiber oscillator is depicted in Fig. 1. It consists of a HNLF for parametric amplification and two WDMCs for wavelength-selective input/output coupling. The operation of the system is as follows.

A strong CW pump wave enters the system at (a) and is passed from port (i) to port (iii) of WDMC1 with high efficiency. Upon propagation through the HNLF, a signal and an idler wave are generated at wavelengths determined by the phase matching condition in the fiber. The majority of the generated idler wave is transmitted in WDMC2 from port (i) to port (iii) and is the target output of the FOPO. On the other hand, the majority of the signal wave is passed from port (i) to port (iv) and thus remains in the oscillator. A linear wavelength-independent loss is applied at point (c) modeling the accumulated effect of splice losses throughout the resonator. WDMC1 finally combines this circulating signal with a new pump wave, which thus becomes the input to the HNLF for the following round trip and so on. Eventually, the recirculation of the sideband within the FOPO cavity leads to its power buildup until the losses of the system counteract its gain. A high sideband power within the oscillator is favorable since it mediates the conversion of energy to the idler output, therefore increasing the CE of the FOPO [1]. Note that a part of the unconverted pump is ejected at port (iii) of WDMC2, while the remainder of the pump is dropped from the oscillator through port (iv) of WDMC1. Two phase modulators are also included in the system to ensure the resonance of the cavity for the circulating signal and the correct relative phase between pump, signal and idler waves before the HNLF to guarantee parametric amplification, as discussed in more detail below.

\section{NUMERICAL MODEL}

The FOPO thus consists of two main subsystems, the WDMCs and the HNLF, which are modeled separately as discussed in the following.

The WDMCs are considered as $2 \times 2$ linear fiber couplers and are modeled using a scattering matrix representation (SMR). Given input fields at ports (i) and (ii) of a WDMC the output fields at ports (iii) and (iv) are found by [26]:

$$
\left(\begin{array}{l}
U^{(i i i)}(f) \\
U^{(i v)}(f)
\end{array}\right)=\left(\begin{array}{cc}
\sin (g(f)) & i \cos (g(f) \\
i \cos (g(f) & \sin (g(f))
\end{array}\right)\left(\begin{array}{c}
U^{(i)}(f) \\
U^{(i i)}(f)
\end{array}\right),
$$

where $U(f)$ is the spectral amplitude at frequency $f$ of the wave under consideration. The function $g(f)$ is obtained by the coupled mode theory that describes the WDMCs $[26,27]$ and determines the transmittance window for each output port. In particular, $g(f)$ is chosen such that the transmittance is maximum between ports (i) and (iii) at a desired wavelength $\lambda_{1}$ and equivalently between (i) and (iv) at a second wavelength $\lambda_{2}$. For the sake of clarity, such a system will be denoted by $\operatorname{WDMC}\left(\lambda_{1}, \lambda_{2}\right)$ hereafter. The SMR is also used to model a frequency-independent round trip loss $\alpha$ (in $\mathrm{dB}$ ) at position (c) in the resonator. Whenever an input port (i) or (ii) is empty, random quantum noise at the level of one photon per frequency mode is injected at this position to provide a consistent noise floor.

The main process of parametric amplification is degenerate FWM where a pump wave at angular frequency $\omega_{p}$ is propagated through the nonlinear fiber resulting in the amplification of the signal wave at the lower angular frequency $\omega_{s}$ and of the idler wave at a higher frequency $\omega_{i}$. Because of energy conservation, the signal and idler have equal frequency separation $\Omega$ from the pump, i.e., $\omega_{p}-\omega_{s}=\omega_{i}-\omega_{p}=\Omega$. If the signal and idler reach sufficiently high powers, further higher order sidebands at frequencies $\omega_{p} \pm 2 \Omega, \omega_{p} \pm 3 \Omega$ etc. can also be created. Since these waves have been shown to create instabilities in FOPOs we include sidebands up to the third order in our model. The nonlinear propagation is described by the BNLSE [17] for 7 frequency bands, $l=-3,-2, \ldots, 3$,

$$
\begin{aligned}
\frac{d A_{l}}{d z}= & \mathrm{i} \sum_{n \geq 2} \frac{\beta_{n}}{n !}\left(\mathrm{i} \frac{\partial}{\partial t}-l \Omega\right)^{n} A_{l}+\mathrm{i} \gamma_{l}\left(1+\frac{\mathrm{i}}{\omega_{l}} \frac{\partial}{\partial t}\right) \times \\
& \sum_{l+m=n+k} A_{k} A_{m}^{*} A_{n}\left\{1-f_{r}+f_{r} \tilde{h}[(m-l) \Omega]\right\}
\end{aligned}
$$

where $A_{l}$ is the envelope function of the wave in band $l, \beta_{n}$ are the Taylor expansion coefficients of the propagation constant $\beta$ calculated at the pump frequency (in band $l=0$ ), $\gamma_{l}$ and $\omega_{l}$ are the nonlinear coefficient and central frequency of each band respectively, $f_{r}$ is the fractional contribution of the 
Raman nonlinearity, $\tilde{h}(\omega)$ is the Fourier transform of the Raman response function at frequency $\omega$. Note that $\tilde{h}(0)=1$ due to the normalization of the Raman response function. The details of the derivation of the BNLSE, its validity and computational efficiency have been discussed in [17]. The second sum in Eq. (2) runs over all indices $k, m, n=-3, \ldots, 3$ such that the corresponding FWM process conserves energy, i.e., $l+m=n+k$. The spectral position of the bands is found by solving the phase matching condition with respect to $\Omega$ [28],

$$
\beta\left(\omega_{0}+\Omega\right)+\beta\left(\omega_{0}-\Omega\right)-2 \beta\left(\omega_{0}\right)+2 \gamma_{0} P=0,
$$

and thus the central frequency of band $l$ is $\omega_{l}=\omega_{0}+l \Omega$. Note that in Eq. (2) we have assumed that all fields are linearly polarized in the same direction and counter-propagating waves are neglected. The BNLSE is solved using an adaptive step-size symmetrized split-step Fourier method [28].

The FOPO is then simulated by iterating over many round trips the sequence of WDMC1, BNLSE, WDMC2, and loss until the output power of the idler at point (d) in Fig. 1 converges. Two additional phases are applied to the propagating fields at every round trip, as indicated in the figure. Before WDMC1, a phase shift $\left(\mathrm{PM}_{2}\right)$ is applied to all propagating bands to emulate length-tuning of the fiber ring resonator. In the presence of a seeded signal at the input of port (i) of WDMC1, this phase shift is used such that the signal seed and the signal circulating in the resonator are added constructively in WDMC1. Additionally, after WDMC1 another phase shift $\left(\mathrm{PM}_{1}\right)$ is applied to the idler wave such that pump, signal and idler are in phase for maximum parametric amplification, i.e. $\phi_{s}+\phi_{i}-2 \phi_{p}=\pi / 2$ where $\phi_{s, i, p}$ are the phases of signal, pump, and idler, respectively [1]. The phase shifts $\mathrm{PM}_{1}$ and $\mathrm{PM}_{2}$ are adapted dynamically at every round trip in the simulation but are found to reach a stationary state. Our simulated steady-state results, therefore, correspond to experimental situations where optimized but constant phase shifts are applied. We also note that the second phase shift, $\mathrm{PM}_{2}$, is only required if there is a (small) amount of idler power circulating in the resonator but it is not needed in situations where the idler wave is fully ejected from the FOPO at WDMC2.

The algorithm to solve the model is written in the Python 3.6 programming language while making use of multiple libraries from the scientific Python stack [29]. The source code used to model the system is available under the BSD 3-Clause License at [30].

The fiber considered for the system is an $18 \mathrm{~m}$ long LMA5-PM fiber, which is a silica-based photonic crystal fiber by NKT Photonics. While the fiber is slightly birefringent, for the purposes of this study we only consider light of polarization along its slow axis. The Taylor expansion coefficients of the propagation constants are given by $\beta_{3}=6.756 \cdot 10^{-2} \mathrm{ps}^{3} / \mathrm{km}, \beta_{4}=-1.002 \cdot 10^{-4}$ $\mathrm{ps}^{4} / \mathrm{km}, \beta_{5}=3.671 \cdot 10^{-7} \mathrm{ps}^{5} / \mathrm{km}$ at a zero dispersion wavelength (ZDW) of $\lambda_{d}=1051.85 \mathrm{~nm}$ and the nonlinear coefficient at the ZDW is $\gamma=10 \mathrm{~W}^{-1} \mathrm{~km}^{-1}$ [31]. Because the material of the fiber is silica the Raman response function described in [32] is used. The spectral position of the frequency bands is determined by solving Eq. (3) for the selected pump frequency and power and the spectral width of the bands is set large enough to avoid boundary condition errors stemming from the Fourier transforms. Finally, fiber attenuation is negligible compared to the other losses per round trip in the FOPO.

Using the notation of Sec. 2, the WDMCs are set as WDMC1 $(1048,1204)$ and WDMC2 $(928,1204)$ (wavelengths in $\mathrm{nm}$ ). This configuration leads to the longer parametric wavelength (signal) oscillating within the fiber cavity and the shorter

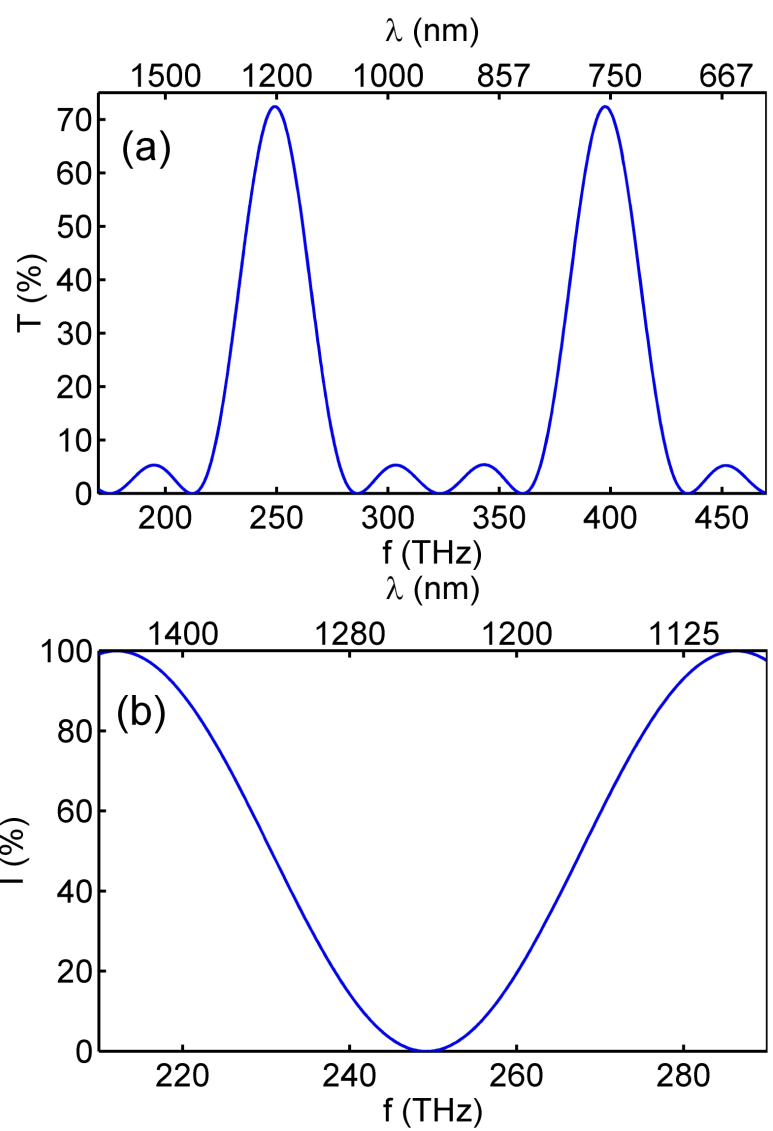

Fig. 2. (a) Frequency/wavelength dependent transmission per round trip of the FOPO and (b) insertion efficiency of WDMC1.

(idler) forming the desired output. The splice loss at point (c) within Fig. 1 is set to $1.4 \mathrm{~dB}$. The combination of the WDMC transmission and the loss result in the wavelength-dependent transmission per round trip inside the FOPO and the insertion efficiency of the input coupler WDMC1 as shown in Fig. 2. The position of the frequency bands considered in the following for pump wavelengths between $1046 \mathrm{~nm}$ and $1050 \mathrm{~nm}$ at $6 \mathrm{~W}$ pump power and their corresponding cavity transmission and insertion efficiency are given in Table 1.

For each set of parameters the FOPO is simulated over a large number of round trips and the output power is calculated as the average of the last $50 \%$ oscillations (after convergence is reached). Numerically we find that low pump powers require a larger number of oscillations until convergence is achieved whereas higher powers converge much more quickly. The CE of the FOPO is defined as the ratio of the time-averaged output power at point (d) and the input power at point (a),

$$
C E=\frac{\left\langle P_{(d)}\right\rangle}{P_{(a)}},
$$

and the equivalent RIN is calculated as

$$
R I N=10 \log \left(\frac{1}{\Delta f} \frac{\sigma_{P}^{2}}{\left\langle P_{(d)}\right\rangle^{2}}\right),
$$

where $\Delta f$ is the frequency grid resolution (and thus the simulated time window is $1 / \Delta f$ ) and $\sigma_{P}$ the standard deviation of 
the output power per round trip from the mean power $\langle P\rangle$. The power $P_{(d)}$ is calculated only at the desired output band $(l=1)$.

\section{SIMULATION RESULTS}

\section{A. Frequency Resolution}

We first investigate the build-up of power in the FWM sidebands as a function of the number of cavity round trips. Fig. 3(a) shows the idler output power at point (d) in the schematic of Fig. 1 averaged over the time window used in the simulation of the BNLSE versus round trip number. Initially, no signal and idler waves are present in the resonator apart from the fundamental quantum noise (of order one photon per frequency channel). Next, because of the strong FWM gain provided by the pump, signal and idler waves are quickly building up from their noise value over several tens of round trips and thus the idler output power increases. Pump depletion then starts to limit the FWM gain and a stationary output is achieved when the round trip gain of the signal and idler sidebands is compensated by the round trip losses as given above in Table 1.

However, Figure 3(a) shows that the achieved stationary state depends strongly on the frequency grid spacing $\Delta f$ used in the BNLSE simulation or, equivalently, on the number of frequency grid points $N_{g}$. In each case, the total width of the simulated frequency range in each band is held constant at $5 \mathrm{THz}$, such that $N_{g} \times \Delta f=5 \mathrm{THz}$. For large $\Delta f$ (very few grid points) the output power is high (around $3 \mathrm{~W}$ for the parameters of the figure) and very stable. For decreasing $\Delta f$ (increasing number of grid points) the predicted output drops and the idler output becomes noisy. This is shown more quantitatively in Figures $3(\mathrm{c}, \mathrm{d})$ which depict the mean stationary output power and RIN versus $\Delta f$ in the range from $1 \mathrm{GHz}$ to $1 \mathrm{THz}$ (number of grid points $N_{g}=2^{2}$ to $2^{12}$ ). At large $\Delta f$, the RIN is determined by the quantum noise floor at $-189 \mathrm{~dB} / \mathrm{Hz}$ at $3 \mathrm{~W}$ power for this system. By decreasing $\Delta f$, we see that the simulation results converge for $\Delta f \lesssim 50 \mathrm{GHz}$. This behavior is explained by the idler output spectra shown in Fig. 3(b): for large $\Delta f$ only a single frequency grid point is within the gain bandwidth of the FOPO, which thus leads to stable CW output. Only if the gain bandwidth is resolved on the frequency grid, a realistic model of the FOPO is achieved which properly describes the generation of a finite bandwidth idler and the intensity noise that is generated through the beating of its individual frequency components. Figures $3(\mathrm{c}, \mathrm{d})$ also show the output power and RIN as a function of the frequency grid spacing for higher input power of $12 \mathrm{~W}$. In this case convergence is achieved at slightly larger values of $\Delta f$ because of the wider gain bandwidth at higher powers. For the simulations in the following sections we will use a grid size of $N_{g}=2^{10}$ and grid spacing of $\Delta f=4.9 \mathrm{GHz}$. We also emphasize that the widths of the frequency grids must be chosen wide enough to accommodate all generated frequency components. In our simulations this is ensured by checking for errors at the grid boundaries at every oscillation.

We note that this result implies that simulations of FOPOs based on three coupled amplitude equations for pump, signal, and idler will generally not produce correct predictions. We also emphasize the importance of using the BNLSE for these simulations, instead of a single NLSE equation that covers the full spectral range of all sidebands. For $N_{g}=2^{10}$ and 7 frequency bands to include potential cascaded FWM sidebands, our simulations overall include 7168 complex amplitudes, whereas a single NLSE covering the same frequency span at the same resolution would require over 57,000 frequency amplitudes. The
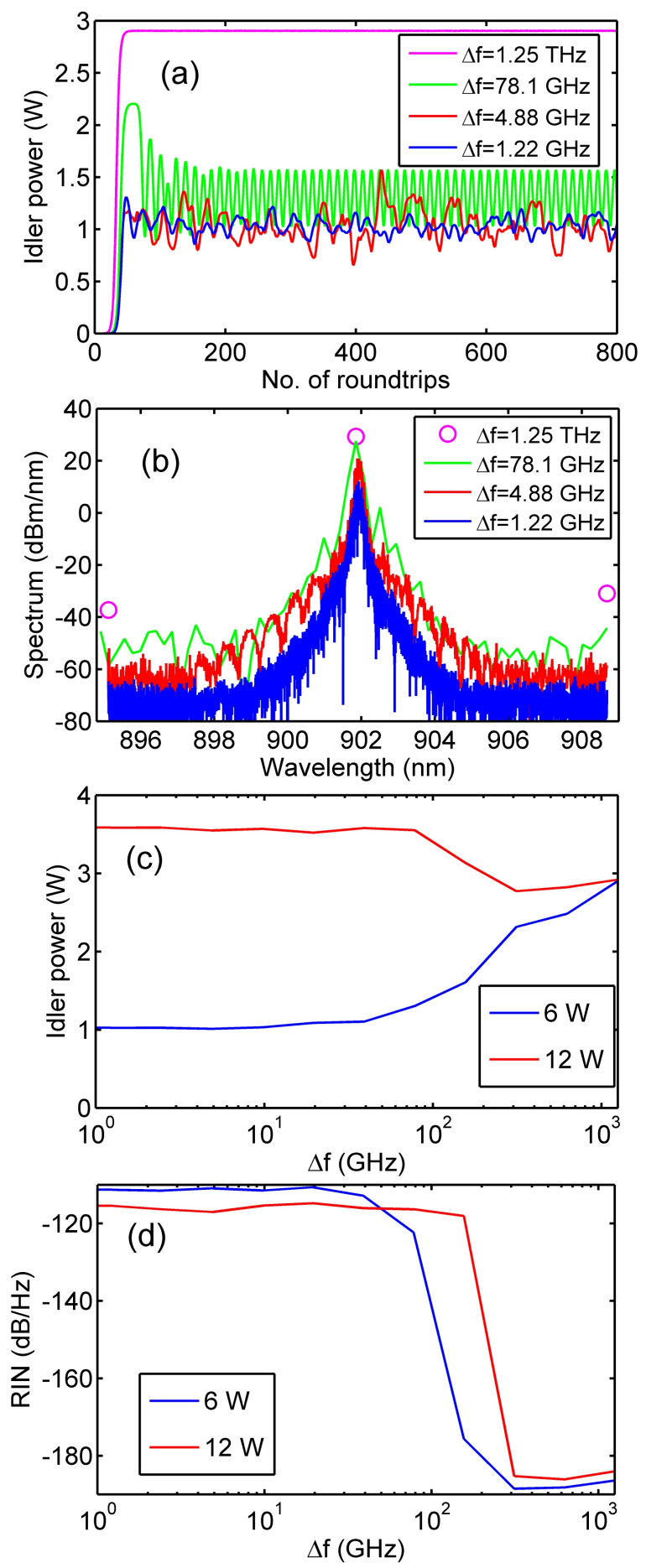

Fig. 3. (a) Idler output power vs number of round trips for different frequency grid spacings $\Delta f$. (b) Corresponding idler spectra. Fore clarity, the curves for $\Delta f=78.1,4.88,1.22 \mathrm{GHz}$ have been offset by $-10,-20,-30 \mathrm{dBm} / \mathrm{nm}$, respectively. (c) Stationary idler output power and (d) RIN versus $\Delta f$ for pump powers of $6 \mathrm{~W}$ and $12 \mathrm{~W}$. The pump wavelength is $1046 \mathrm{~nm}$ for all figures, and the pump power is $6 \mathrm{~W}$ for $(\mathrm{a}, \mathrm{b})$.

BNLSE/NLSE then needs to be solved for hundreds or even thousands of round trips to calculate a full FOPO dynamics as in Fig. 3(a). 
Table 1. Central wavelengths of frequency bands for different pump wavelengths (band $l=0)$ and spectral transmittance/insertion of signal wavelength at band $l=-1$.

\begin{tabular}{|c|c|c||c||c|c|c||c|c|}
\hline \multicolumn{7}{|c||}{ Central wavelength of band $l(\mathbf{n m})$} & Transmittance \& Insertion of signal (\%) \\
\hline-3 & -2 & -1 & 0 & 1 & 2 & 3 & $T_{S}$ & $I_{S}$ \\
\hline \hline 2017 & 1540.3 & 1245.9 & 1046 & 901.4 & 791.9 & 706.1 & 61.8 & 12 \\
\hline 1861 & 1478 & 1225.7 & 1047 & 913.8 & 810.6 & 728.4 & 69.4 & 3.4 \\
\hline 1714.8 & 1414.8 & 1204.1 & 1048 & 927.7 & 832.2 & 754.6 & 72.45 & 0 \\
\hline 1574.8 & 1349.3 & 1180.4 & 1049 & 943.9 & 858 & 786.4 & 68.45 & 4.45 \\
\hline 1435.3 & 1278.9 & 1153.2 & 1050 & 963.8 & 890.6 & 827.8 & 54.75 & 20.2 \\
\hline
\end{tabular}

\section{B. Unseeded FOPO}
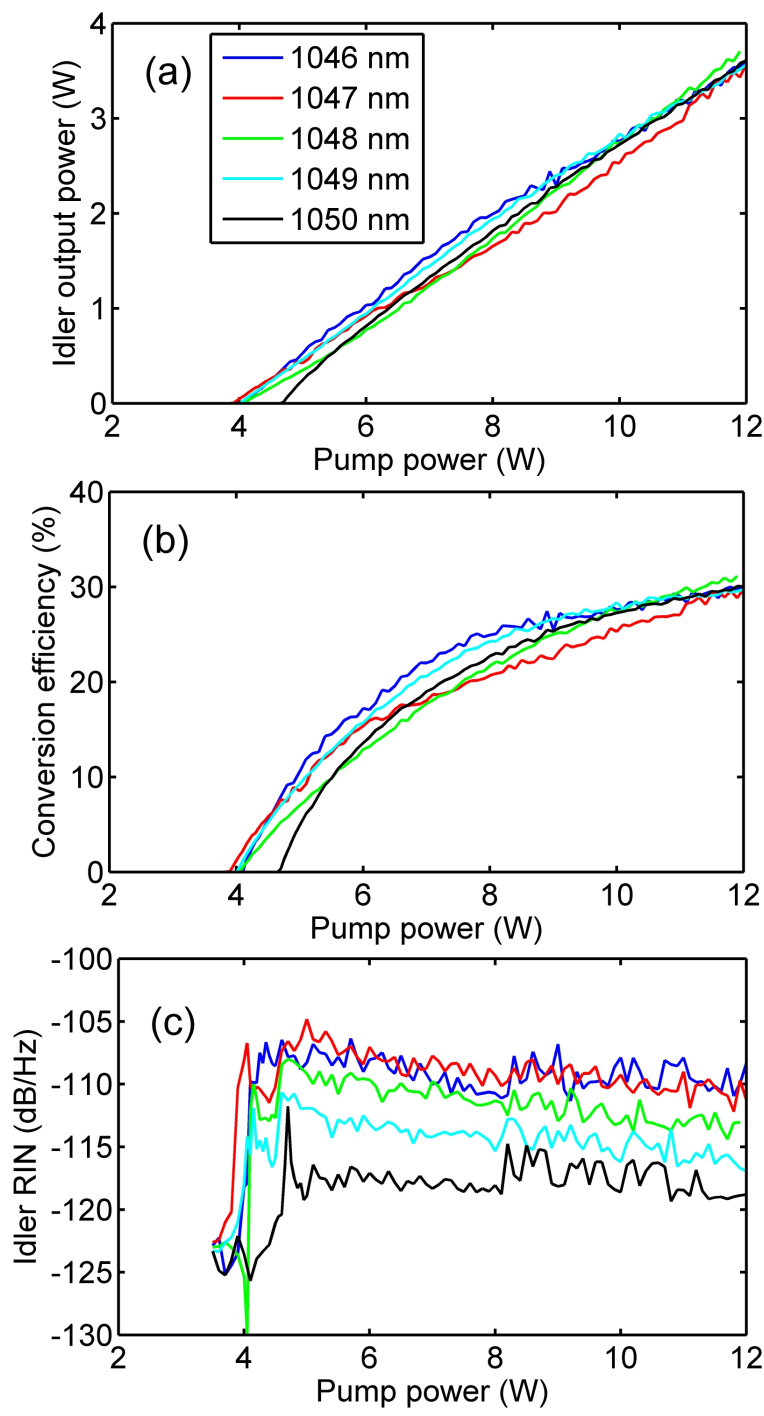

Fig. 4. (a) Stationary idler power, (b) conversion efficiency, and (c) RIN vs pump power at different pump wavelengths for the fiber OPO depicted in Fig. 1 when only the pump wave is present at the input. All parameters are given in the text.

We next consider the behavior of such a FOPO system in the stationary regime, i.e., after a large number of round trips where the mean idler output power and its variance have reached sta- tionary values. The idler power, CE, and RIN versus pump power for pump wavelengths from 1046 to $1050 \mathrm{~nm}$ are presented in Fig. 4.

Our numerical simulations find threshold pump powers of approximately $4.10 \mathrm{~W}$ (1046 nm pump), 3.90 W (1047 nm pump), $4.05 \mathrm{~W}$ (1048 nm pump), $4.00 \mathrm{~W}$ (1049 nm pump), and $4.65 \mathrm{~W}$ (1050 nm pump), respectively, where the largest threshold powers correspond to the parameters with the largest signal round trip loss (cf. Table 1) as expected. We note that these threshold values are in excellent agreement with experimental results [33] reported as $4.1 \mathrm{~W}$ for an idler wave at $931 \mathrm{~nm}$ (corresponding to a pump wavelength of $\sim 1048 \mathrm{~nm}$ ). By contrast, the threshold expected at this wavelength based on a simple coupled-amplitude equation [1] which also overestimates the nonlinear factor $\gamma$ by neglecting the Raman contribution [17],

$$
P_{\text {th }}=(\gamma L)^{-1} \cosh ^{-1}\left(1 / \sqrt{T_{S}}\right)
$$

where $T_{S}$ is the round trip transmission of Table 1 , gives a significantly lower value of $3.3 \mathrm{~W}$. However, we note that while our model captures the frequency resolved dynamics of the system, we still assume monochromatic $(\mathrm{CW})$ pumping and thus ignore the pump laser line width or frequency dithering that is applied experimentally to avoid stimulated Brillouin scattering.

For pump powers above the threshold we find an approximately linear increase of idler output power with pump power that is very similar for all simulated pump wavelengths. The conversion efficiency of pump power into idler output power increases from 0 at threshold to $\sim 30 \%$ at $12 \mathrm{~W}$ pump power. Note that in an ideal lossless parametric process where the pump power is fully converted into signal and idler, the maximum CE for an idler at $928 \mathrm{~nm}$ with a $1048 \mathrm{~nm}$ pump would be $56.5 \%$ as determined by the difference in idler and signal photon energies.

The idler RIN is depicted in Fig. 4(c). Below threshold, the numerical RIN value obtained from our noise floor is $-127 \mathrm{~dB} / \mathrm{Hz}$. Above threshold, the idler noise is in fact increased beyond this white noise for all pump wavelengths. As discussed in the previous section, the idler wave contains a bandwidth of frequencies which compete for the parametric gain, and their random relative phase leads to strong interference effects in the idler power in the time domain, thus affecting the RIN. Moreover, because of anomalous fiber dispersion of the oscillating signal wave, the signal wave is also subject to modulation instability which subsequently affects the generation of the idler wave [24].

However, there are significant differences in the idler RIN for pumping at different wavelengths, Fig. 4(c), where despite the comparable signal power, as discussed above, the RIN reduces by approximately $10 \mathrm{~dB}$ when the pump wavelength changes from $1046 \mathrm{~nm}$ to $1050 \mathrm{~nm}$. We attribute this to the different 
parametric gain bandwidths for the different pump wavelengths. If we estimate the idler gain bandwidth as the angular frequency range $\Delta \omega_{\text {idler }}$ where the phase mismatch over the fiber length of $L=18 \mathrm{~m}$ is less than $\pi$, we find to lowest order in the Taylor expansion of the propagation constant

$$
\Delta \omega_{\text {idler }}=\frac{\pi}{L \beta_{3}\left(\omega_{p}-\omega_{d}\right)\left(\omega_{i}-\omega_{p}\right)}
$$

where $\omega_{d}=2 \pi c / \lambda_{d}$ is the angular frequency of the ZDW. For our fiber parameters, this yields an idler gain bandwidth of $0.385 \mathrm{~nm}$ at $1046 \mathrm{~nm}$ pumping and $2.55 \mathrm{~nm}$ at $1050 \mathrm{~nm}$, an increase of bandwidth by a factor of 6.54 or $8.2 \mathrm{~dB}$. Note that for uncorrelated amplitude and phase noise between frequency components at constant average output power, Eq. (5) suggests that RIN reduces linearly with increasing bandwidth, which matches well with our observation of $\sim 10 \mathrm{~dB}$ improved RIN for an increase of bandwidth by $8.2 \mathrm{~dB}$.

\section{Seeded FOPO}

As discussed above, the large RIN of the oscillator is attributed to the spectral width of the oscillating parametric sidebands. Therefore, we will now consider the effect of a continuous wave (i.e., narrow band) input seed to the oscillator at a wavelength at the center of the frequency band $l=-1$ (signal wave). The seed power is set at $P_{S}=100 \mathrm{~mW}$ at point (a) of the system (see Fig. 1 ), of which a fraction $I_{S}$ is coupled into the oscillator through WDMC1 depending on the seed wavelength as shown in Table 1. Because of the configuration of the FOPO, a signal wavelength of $1048 \mathrm{~nm}$ cannot be coupled into the cavity in this way and therefore is not considered in the following. We also emphasize again that in the seeded configuration, two independent phase controllers are required inside the oscillator (labeled $\mathrm{PM}_{1}$ and $\mathrm{PM}_{2}$ in Fig. 1) to ensure the correct relative phase between pump, signal, and idler wave for parametric amplification, and to make the oscillator resonant for the signal wave frequency, respectively.

Figure 5(a) shows the idler output power versus pump power for four different pump wavelengths. No well defined threshold power is found in this case, but an idler wave is generated already at pump powers well below $1 \mathrm{~W}$. For increasing pump power, the idler power first increases nonlinearly at all pump wavelengths up to around $3 \mathrm{~W}$ pump power, then experiences a regime of slow, but approximately linear increase up to around 4$5 \mathrm{~W}$, and finally exhibits strong linear increase for larger powers. A magnified version of the region below $6 \mathrm{~W}$, Fig. 5(b), shows that this latter regime corresponds to pump powers above the threshold of unseeded operation. The comparison with the results from the previous section (dashed curves) indicates that above the unseeded oscillator threshold, the output power is approximately the same in both cases of an unseeded and a weakly seeded FOPO and thus the addition of a seed signal wave brings no advantage to the FOPO output.

The CE of the seeded FOPO is plotted in Fig. 5(c). We see that conversion efficiencies up to around $10 \%$ can be achieved below the threshold power. There is again a nonlinear rise of the $\mathrm{CE}$ at low pump powers, which seems to saturate around the threshold powers before the CE curves follow the same behavior as the unseeded results from Fig. 4. Similar observations hold for the RIN of the output idler wave, Fig. 5(d). Below the thresholds, the RIN is decreasing with increasing power as expected for monochromatic signals where at higher powers the signal-tonoise ratio decreases. After some transition region around the threshold powers, the RIN takes on the same values as in the
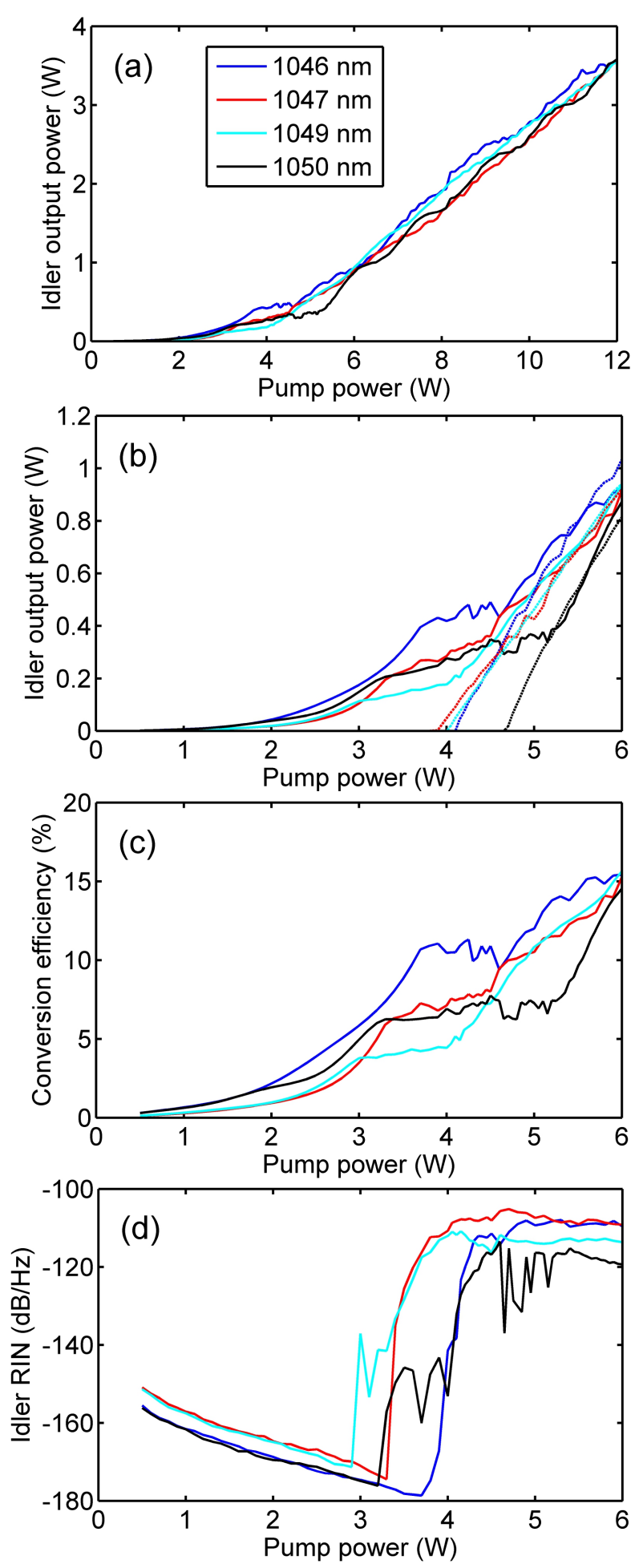

Fig. 5. Results of the FOPO when a weak signal seed wave of $100 \mathrm{~mW}$ power is injected in addition to the pump field. (a) Stationary idler output power, (b) zoom into the threshold region of idler power (solid) and comparison with the unseeded FOPO (dashed), (c) conversion efficiency, (d) RIN vs pump power for different pump wavelengths. All other parameters are given in the text. 
unseeded configuration, indicating that despite the presence of a monochromatic seed signal, a broad bandwidth of signal and idler frequencies are contributing to the output power in this regime.

\section{SUMMARY AND CONCLUSIONS}

We presented a numerical model of a fiber optical parametric oscillator ring with WDMCs for input and output coupling. The model exploits the banded nonlinear Schrödinger equation [17] to spectrally resolve the gain bandwidth of the generated signal and idler waves. It was shown that such a fine spectral resolution is essential to achieve reliable results. Specifically, we demonstrated that the model provides pump threshold powers in agreement with experimental data [33] and also predicts large intensity noise of the generated idler wave. None of these results, threshold power, output idler power and noise, can be achieved with a simplified model of coupled amplitude equations where pump, signal, and idler waves are treated as CW.

The output powers were found to behave similarly for a range of pump wavelengths close to the ZDW which correspond to over $100 \mathrm{~nm}$ variation of the signal wavelength and over 60 $\mathrm{nm}$ variation of the idler wavelength. The RIN, on the other hand, varies for different pump wavelengths and while the RIN is generally very high for the chosen system (of order 110 to $120 \mathrm{~dB} / \mathrm{Hz}$ ) we found that larger gain bandwidths, and thus pumping closer to the ZDW, in general leads to less noisy FOPO output.

Finally, we investigated the dynamics of seeded FOPOs where a weak monochromatic signal wave is additionally injected into the fiber resonator. This allows for the generation of a low-noise idler output with conversion efficiencies up to $\sim 10 \%$ below the threshold pump power of the unseeded FOPO. For pump powers above the threshold, however, also in the seeded configuration a broad bandwidth of signal and idler frequencies contribute to the system dynamics and the results of the seeded FOPO matched those of the unseeded one in this regime.

In conclusion, while WDMC-based FOPOs can operate over a large bandwidth and exhibit relatively high $\mathrm{CE}$, they also exhibit large intensity noise which, at high powers, even prevails if the oscillator is seeded with a narrow linewidth signal.

\section{FUNDING}

EPSRC Centre for Doctoral Training in Next Generation Computational Modelling (EP/L015382/1).

\section{ACKNOWLEDGMENTS}

The authors acknowledge the use of IRIDIS High Performance Computing facility and associated services at the University of Southampton in the completion of this work.

The data used in this paper is openly available at https://doi.org/10.5258/SOTON/D0751.

\section{REFERENCES}

1. M. E. Marhic, Fiber Optical Parametric Amplifiers, Oscillators and Related Devices (Cambridge University Press, Cambridge, 2008).

2. M. E. Marhic, N. Kagi, T.-K. Chiang, and L. G. Kazovsky, "Broadband fiber optical parametric amplifiers," Opt. Lett. 21, 573-575 (1996).

3. J. S. Y. Chen, S. G. Murdoch, R. Leonhardt, and J. D. Harvey, "Effect of dispersion fluctuations on widely tunable optical parametric amplification in photonic crystal fibers," Opt. Express 14, 9491-9501 (2006).
4. E. A. Zlobina, V. Mishra, S. I. Kablukov, S. P. Singh, S. K. Varshney, and S. A. Babin, "Specifics of short-wavelength generation in a continuous wave fiber optical parametric oscillator," Laser Phys. Lett. 13, 115106 (2016).

5. M. Nakazawa, K. Suzuki, and H. A. Haus, "The Modulational Instability Laser-part I: Experiment," IEEE J. Quantum Electron. 25, 2036-2044 (1989).

6. M. Nakazawa, K. Suzuki, H. Kubota, and H. A. Haus, "The Modulational Instability Laser-part li: Theory," IEEE J. Quantum Electron. 25, 20452052 (1989).

7. S. Saito, M. Kishi, and M. Tsuchiya, "Dispersion-flattened-fibre optical parametric oscillator for wideband wavelength-tunable ps pulse generation," Electron. Lett. 39, 2003 (2003).

8. Y. Zhou, K. K. Y. Cheung, S. Yang, P. C. Chui, and K. K. Y. Wong, "UltraWidely tunable, narrow linewidth picosecond fiber-optical parametric oscillator," IEEE Photonics Technol. Lett. 22, 1756-1758 (2010).

9. L. Zhang, S. Yang, P. Li, X. Wang, D. Gou, W. Chen, W. Luo, H. Chen, M. Chen, and S. Xie, "An all-fiber continuously time-dispersion-tuned picosecond optical parametric oscillator at $1 \mu \mathrm{m}$ region." Opt. Express 21, 25167-25173 (2013).

10. L. Zhang, S. Yang, X. Wang, D. Gou, H. Chen, M. Chen, and S. Xie, "High-efficiency all-fibre optical parametric oscillator based on photonic crystal fibres pumped by ytterbium-doped fibre laser," Electron. Lett. 50, 624-626 (2014).

11. E. A. Zlobina and S. I. Kablukov, "Fiber optical parametric oscillators," Optoelectronics, Instrumentation Data Process. 49, 363-382 (2013).

12. A. Gershikov, E. Shumakher, A. Willinger, and G. Eisenstein, "Fiber parametric oscillator for the $2 \mu \mathrm{m}$ wavelength range based on narrowband optical parametric amplification." Opt. Lett. 35, 3198-3200 (2010).

13. E. A. Zlobina, S. I. Kablukov, and S. A. Babin, "All-PM CW fiber optical parametric oscillator," Opt. Express 24, 25409-25414 (2016).

14. M. E. Marhic, K. K.-Y. Wong, L. G. Kazovsky, and T.-E. Tsai, "Continuous-wave fiber optical parametric oscillator." Opt. Lett. 27, 1439-1441 (2002).

15. W. Q. Zhang, J. E. Sharping, R. T. White, T. M. Monro, and S. Afshar V., "Design and optimization of fiber optical parametric oscillators for femtosecond pulse generation," Opt. Express 18, 17294-17305 (2010).

16. E. S. Lamb, S. Lefrancois, M. Ji, W. J. Wadsworth, X. Sunney Xie, and F. W. Wise, "Fiber optical parametric oscillator for coherent anti-Stokes Raman scattering microscopy," Opt. Lett. 38, 4154-4157 (2013).

17. I. Begleris and P. Horak, "Frequency-banded nonlinear Schrödinger equation with inclusion of Raman nonlinearity," Opt. Express 26, 2152721536 (2018).

18. Y. Li, L. Qian, D. Lu, and D. Fan, "Widely tunable femtosecond fiber optical parametric oscillator," Opt. Commun. 267, 491-497 (2006).

19. H. Cheng, Z. Luo, C. Ye, Y. Huang, C. Liu, and Z. Cai, "Numerical modeling of mid-infrared fiber optical parametric oscillator based on the degenerated FWM of tellurite photonic crystal fiber," Appl. Opt. 52, 525-529 (2013).

20. H. Bai, X. Yang, Y. Wei, and S. Gao, "Broadband mid-infrared fiber optical parametric oscillator based on a three-hole suspended-core chalcogenide fiber," Appl. Opt. 55, 515-521 (2016).

21. J. Zhao, B. Luo, W. Pan, L. Yan, X. Zou, J. Ye, H. Zhu, N. Li, and Z. Chen, "Output characterization of a fiber optic parametric oscillator based on multiple four-wave mixing," Appl. Opt. 54, 7884-7888 (2015)

22. I. Begleris and P. Horak, "Cascade simulations of unidirectional fiber optical parametric oscillators," in 2017 International Conference on Numerical Simulation of Optoelectronic Devices (NUSOD), (Copenhagen, 2017), pp. 169-170.

23. G. Rustad, G. Arisholm, and Ø. Farsund, "Effect of idler absorption in pulsed optical parametric oscillators," Opt. Express 19, 2815-2830 (2011).

24. K. S. Yeo, F. R. M. Adikan, M. Mokhtar, S. Hitam, and M. A. Mahdi, "Continuous wave tunable fiber optical parametric oscillator with doublepass pump configuration," Appl. Phys. B: Lasers Opt. 110, 353-357 (2013).

25. K. S. Yeo, M. H. A. Bakar, F. R. M. Adikan, and M. A. Mahdi, "Widely 
Tunable Fiber Optical Parametric Oscillators with Idler Removal Filter," IEEE Photonics J. 9, 1400109 (2017).

26. G. Keiser, Optical fiber communications, McGraw-Hill series in electrical engineering: Communications and information theory (McGraw-Hill Higher Education, Singapore, 2011), 3rd ed.

27. K. Okamoto, Fundamentals of optical waveguides (Elsevier, 2005), 2nd ed.

28. G. P. Agrawal, Nonlinear fiber optics (Academic Press, 2013), 5th ed.

29. K. J. Millman and M. Aivazis, "Python for scientists and engineers," Comput. Sci. Eng. 13, 9-12 (2011).

30. I. Begleris and P. Horak, "Computational-Nonlinear-Optics-ORC/Singlemode- FOPO: High resolution SM-FOPO with BNLSE," (2018).

31. E. A. Zlobina, S. I. Kablukov, and S. A. Babin, "Phase matching for parametric generation in polarization maintaining photonic crystal fiber pumped by tunable Yb-doped fiber laser," J. Opt. Soc. Am. B 29, 19591966 (2012).

32. R. H. Stolen and E. P. Ippen, "Raman gain in glass optical waveguides," Appl. Phys. Lett. 22, 276-278 (1973).

33. E. A. Zlobina, S. I. Kablukov, and S. A. Babin, "High-efficiency CW all-fiber parametric oscillator tunable in $0.92-1 \mu \mathrm{m}$ range," Opt. Express 23, 833-838 (2015). 